\title{
Dynamics and Control of Reactor - Feed Effluent Heat Exchanger Networks
}

\author{
Sujit S. Jogwar*, Michael Baldea ${ }^{\dagger}$ and Prodromos Daoutidis* \\ ${ }^{*}$ Department of Chemical Engineering and Materials Science \\ University of Minnesota, Minneapolis, MN, USA \\ ${ }^{\dagger}$ Praxair, Inc., 175 East Park Drive, Tonawanda, NY, USA
}

\begin{abstract}
In this paper, we analyze the energy dynamics of process networks comprising of a chemical reactor and a feed effluent heat exchanger (FEHE). Using singular perturbation analysis, we show that, in the case of tight energy integration, the energy dynamics of the network evolves over two time scales, with the enthalpy of individual units evolving in the fast time scale and the overall network enthalpy evolving in the slow time scale. We describe a model reduction procedure to derive the non-stiff slow model which can be used for controller design. The theoretical results are illustrated via a simulation case study.
\end{abstract}

\section{INTRODUCTION}

Feed Effluent Heat Exchangers (FEHEs) are key components in the design of heat integrated processes (e.g., [1]). A FEHE transfers the heat available with the hot effluent stream from a chemical reactor to the cold reactor inlet stream, thus leading to a positive feedback of energy. Because of this positive feedback of energy, there is a potential for open-loop instability ([2], [3], [4], [5], [6], [7]) and complex dynamics [8], which in turn demands that an appropriate control system be implemented. A generic set up for the energy integration system with a FEHE is shown in Fig. 1.

The typical control objectives in such networks are controlling the outlet stream composition (a product quality specification), the final effluent temperature (a separation system specification) and the holdup of the network. Several papers ([3], [9], [10]) have focused on this control problem within a multi-loop linear control framework.

In this paper, we develop a generic dynamic analysis of reactor-FEHE networks documenting that in the case of tight energy integration, the energy dynamics of such networks exhibits a two-time scale behavior. We present a model reduction procedure based on singular perturbations to derive non-stiff models in each time scale. We then propose a controller design framework that alleviates the issues typically associated with controllers synthesized based on stiff process models. Finally, the theoretical concepts developed are illustrated via a case study.

\section{Modeling ANd Model Reduction of FEHE-INTEGRATED PROCESSES}

Let us consider a more detailed alternative representation of the network in Fig. 1, shown in Fig. 2, whereby the cold and hot passes of the FEHE (of duty $h_{t r}$ ) are represented separately. To improve dynamic operability and allow for startup strategies, a reactor-FEHE network is usually accompanied

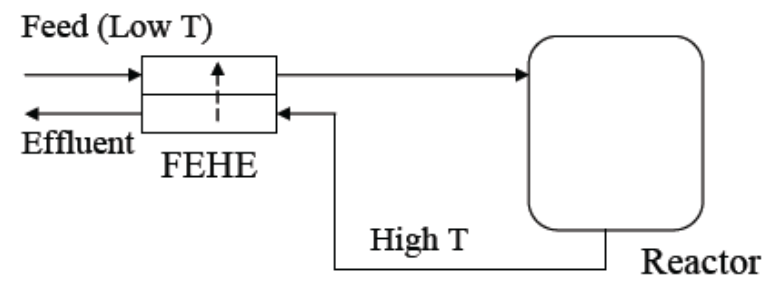

Fig. 1. A heat integrated process with a FEHE (T: Temperature)

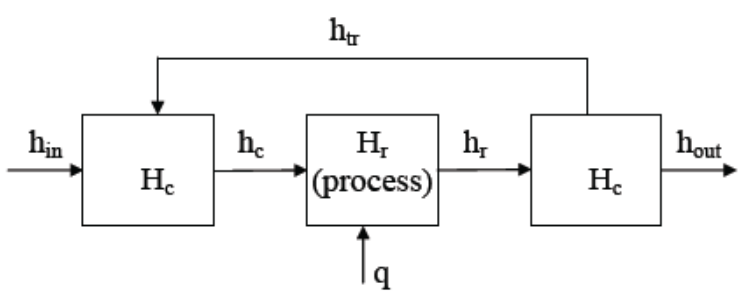

Fig. 2. Abstract representation of the network

by a furnace and/or cooler and a bypass stream. In Fig. 2, the process block contains the reactor and the furnace and/or cooler. Let $h_{\text {in }}, h_{c}, h_{c}$ and $h_{\text {out }}$ be the enthalpy flowrates associated respectively with the inlet, cold leg output, process output and network output streams, and $H_{c}, H_{h}$ and $H_{r}$ the enthalpies of the cold and hot leg of the FEHE, and the enthalpy of the process, respectively. Let $q$ denote the rate of energy generation/consumption for the process (e.g., through the heat of reaction or the furnace/cooler duty).

With the above notation, the energy balance for the units of the network in Fig. 2 takes the form:

$$
\begin{aligned}
\dot{H}_{c} & =h_{i n}-h_{c}+h_{t r} \\
\dot{H}_{r} & =h_{c}-h_{r}+q \\
\dot{H}_{h} & =h_{r}-h_{t r}-h_{\text {out }}
\end{aligned}
$$

We specifically consider the case of an adiabatically operated reactor with moderately exothermic/endothermic reactions (in the case of highly exothermic/endothermic reactions, the adiabatic operation is impractical). The FEHE is designed (e.g., by providing a large heat transfer area) to facilitate large recovery and recycle of energy, leading to tight energy integration. In this case, the energy recycle is large compared to the energy input through the feed 
( $h_{i n, s} / h_{t r, s}=\varepsilon<<1$ where the subscript $s$ denotes the steady state values). The energy input term $q$ is also of comparable magnitude to the energy input of the feed stream $\left(q_{s} / h_{i n, s}=\mathcal{O}(1)\right)$ which follows from the moderate heat effects due to reactions and the large recycled energy leading to small furnace/cooler duties. The internal and external energy flows will be of similar magnitude to those of the energy recycle and the energy input respectively (i.e., $h_{c, s} / h_{t r, s}=\mathcal{O}(1), h_{\text {out }, s} / h_{\text {in }, s}=\mathcal{O}(1)$ ). We can now define the $\mathcal{O}(1)$ steady state ratios $k_{c}=h_{c, s} / h_{t r, s}$ and $k_{r}=h_{r, s} / h_{t r, s}$ and scaled enthalpy flows $u_{c}=h_{c} / h_{c, s}$, $u_{r}=h_{r} / h_{r, s}$ and $u_{t}=h_{t r} / h_{t r, s}$. The model in Eq. (1) then becomes:

$$
\begin{aligned}
\dot{H}_{c} & =h_{i n}+\frac{1}{\varepsilon} h_{i n, s}\left(-k_{c} u_{c}+u_{t}\right) \\
\dot{H}_{r} & =\frac{1}{\varepsilon} h_{i n, s}\left(k_{c} u_{c}-k_{r} u_{r}\right)+q \\
\dot{H}_{h} & =\frac{1}{\varepsilon} h_{\text {in }, s}\left(k_{r} u_{r}-u_{t}\right)-h_{\text {out }}
\end{aligned}
$$

Owing to the presence of the small parameter $\varepsilon$, the above model is in a singularly perturbed form, having the potential to exhibit a dynamic behavior with two time scales. In what follows, we aim to elucidate this behavior and start by defining the fast, "stretched" time scale $\tau=t / \varepsilon$, in which the model of the network becomes:

$$
\begin{aligned}
\frac{d H_{c}}{d \tau} & =\varepsilon h_{i n}+h_{i n, s}\left(-k_{c} u_{c}+u_{t}\right) \\
\frac{d H_{r}}{d \tau} & =h_{i n, s}\left(k_{c} u_{c}-k_{r} u_{r}\right)+\varepsilon q \\
\frac{d H_{h}}{d \tau} & =h_{\text {in,s }}\left(k_{r} u_{r}-u_{t}\right)-\varepsilon h_{\text {out }}
\end{aligned}
$$

In the limiting case $\varepsilon \rightarrow 0$, corresponding to an infinitely high energy recycle, we obtain the following description of the fast dynamics of the network:

$$
\begin{aligned}
\frac{d H_{c}}{d \tau} & =h_{i n, s}\left(-k_{c} u_{c}+u_{t}\right) \\
\frac{d H_{r}}{d \tau} & =h_{i n, s}\left(k_{c} u_{c}-k_{r} u_{r}\right) \\
\frac{d H_{h}}{d \tau} & =h_{i n, s}\left(k_{r} u_{r}-u_{t}\right)
\end{aligned}
$$

In order to focus on the slow dynamics of the network, let us consider the same limit case in the original time scale $t$, obtaining:

$$
\begin{aligned}
0 & =-k_{c} u_{c}+u_{t} \\
0 & =k_{c} u_{c}-k_{r} u_{r} \\
0 & =k_{r} u_{r}-u_{t}
\end{aligned}
$$

which represent a set of quasi-steady state conditions for the fast dynamics (4). Notice that only two of the constraints in (5) are independent, indicating the possibility of a slow component being present in the dynamic behavior of the network. In order to delineate this slow component, we consider the limiting case $\varepsilon \rightarrow 0$ in the original time scale $t$, under the constraints (5). In this case, the terms $z_{1}=$ $\lim _{\varepsilon \rightarrow 0}\left(-k_{c} u_{c}+u_{t}\right) / \varepsilon,-\left(z_{1}+z_{2}\right)=\lim _{\varepsilon \rightarrow 0}\left(k_{c} u_{c}-k_{r} u_{r}\right) / \varepsilon$ and $z_{2}=\lim _{\varepsilon \rightarrow 0}\left(k_{r} u_{r}-u_{t}\right) / \varepsilon$ become indeterminate (remaining, however, finite). With the above considerations, we obtain an expression of the slow energy dynamics of the network in the form of a differential-algebraic equation system:

$$
\begin{aligned}
\dot{H}_{c} & =h_{\text {in }}+h_{\text {in }, s} z_{1} \\
\dot{H}_{r} & =-h_{\text {in }, s}\left(z_{1}+z_{2}\right)+q \\
\dot{H}_{h} & =h_{\text {in }, s} z_{2}-h_{\text {out }} \\
0 & =-k_{c} u_{c}+u_{t} \\
0 & =k_{r} u_{r}-u_{t}
\end{aligned}
$$

The constraints of Eq. (6) can be differentiated and an ODE representation (state space realization) of the DAE system (6) can be obtained once the enthalpy flowrates $u_{c}$, $u_{r}$ and $u_{t}$ are specified via appropriate energy transfer and energy flow correlations in terms of temperature gradients, heat transfer parameters, etc. Note that the order of this state space realization would be at most one. It is however, easy to observe that the overall energy balance for the network

$$
\dot{H}_{\text {total }}=h_{\text {in }}-h_{\text {out }}+q
$$

with $H_{\text {total }}=H_{c}+H_{r}+H_{h}$ is devoid of any large terms, and that $H_{\text {total }}$ therefore only evolves over a slow time horizon. $H_{\text {total }}$ would therefore be an appropriate variable choice to describe the slow dynamics of the network. Note also that the description in Eq. (7) is one-dimensional as stated above.

Remark II.1 The arguments about the magnitude of the energy flow $q$ that were used in the derivations above imply that $q$ is small at steady state. This is not necessarily true during process startup, when a significant amount of energy needs to be accumulated in the process, and in which case the energy input associated with $q$ may be very large.

Remark II.2 The above analysis indicates that the control objectives related to the energy balance of the individual units of the network should be addressed using the large internal enthalpy flows, while the control and, more importantly, optimization of the energy utilization at the level of the entire network should be undertaken using the small energy flows of the network as manipulated inputs. This is contrary to the case of reactor - external heat exchanger network with a large energy throughput, wherein the entire energy dynamics of the network evolve in the fast time-scale [11].

Remark II.3 We can note an analogy of this analysis with the case of material recycle [12], wherein for the case with large material recycle, the dynamics of the individual units evolve in the fast time scale and the dynamics of the overall network evolve in a slow time scale.

\section{CASE STUdy}

We now consider the specific prototype network shown in Fig. 3. It consists of a CSTR, a furnace, and a FEHE with a bypass on the hot stream. We assume that $m$ reactions involving $n$ species are being carried out in the CSTR. 


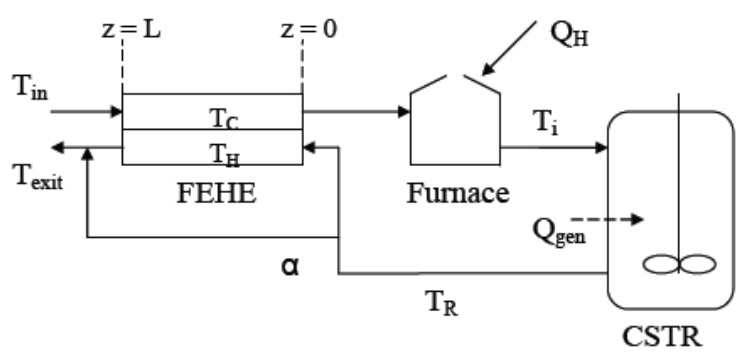

Fig. 3. FEHE, furnace and a CSTR

The hot outlet stream of the reactor is used to partially preheat the cold feed in the FEHE. The reactor input is further heated to the required temperature in the furnace. $\underline{C}$ represents the vector of concentrations of all species and $\underline{C}_{o}$ represents the vector of initial concentrations. $\underline{r}=$ $\left[\begin{array}{llll}r_{1} & r_{2} & \ldots & r_{m}\end{array}\right]^{T}$ represents the vector of reaction rates and $\underline{S}^{n \times m}$ represents the matrix of stoichiometric coefficients. $\Delta H=\left[\begin{array}{llll}\Delta H_{1} & \Delta H_{2} & \ldots & \Delta H_{m}\end{array}\right]^{T}$ represents the vector of heats of reactions. $F_{i n}$ is the volumetric flow rate of the input stream to the network and $F$ is the reactor outlet flow rate; $V, V_{H}, V_{C}$ and $V_{f}$ are the holdups of the reactor, the FEHE hot and cold streams and the furnace respectively. $T_{i n}$ is the cold leg inlet temperature to the FEHE. $T_{H}$ and $T_{C}$ are the hot and cold leg temperatures for the FEHE. $T_{i}$ and $T_{R}$ are the reactor inlet and outlet temperatures and $T_{\text {exit }}$ is the network exit temperature. $Q_{H}$ is the furnace heat duty.

For simplicity, we assume constant heat capacities and densities, no phase change in the FEHE, no heat loses and constant holdups for the FEHE. The dynamic model of the network then takes the form:

$$
\begin{aligned}
d V / d t & =F_{i n}-F \\
d \underline{C} / d t & =F_{i n}\left(\underline{C}_{o}-\underline{C}\right) / V-\underline{\underline{S r}} \\
d T_{R} / d t & =F_{i n}\left(T_{i}-T_{R}\right) / V-\underline{\Delta} H^{T} \underline{r} / \rho c_{p} \\
\partial T_{H} / \partial t & =-v_{H} \partial T_{H} / \partial z-\left(U A / \rho c_{p}\right)\left(T_{H}-T_{C}\right) / V_{H} \\
\partial T_{C} / \partial t & =v_{C} \partial T_{C} / \partial z+\left(U A / \rho c_{p}\right)\left(T_{H}-T_{C}\right) / V_{C}(8) \\
d T_{i} / d t & =F_{i n}\left(T_{C_{(z=0)}}-T_{i}\right) / V_{f}+Q_{H} / \rho c_{p} V_{f}
\end{aligned}
$$

with,

$$
\begin{aligned}
& T_{\text {exit }}=\alpha T_{R}+(1-\alpha) T_{H_{(z=L)}} \\
& T_{H_{z=0}}=T_{R} \\
& T_{C_{z=L}}=T_{i n}
\end{aligned}
$$

where $v_{H}$ and $v_{C}$ are the velocities of the fluid in the hot and cold compartments of FEHE, $L$ is the length of the exchanger, $z$ is the spatial coordinate, and $\alpha$ is a bypass ratio defined as:

$$
\alpha=\frac{\text { flow rate of the bypass stream }}{\text { flow rate of the original stream }(F)}
$$

Comparing this network with the one in Fig. 2, we see that the process block consists of the furnace and the CSTR.
The energy generation/consumption term $q$ is:

$$
q=Q_{H} \pm Q_{g e n}
$$

where $Q_{g e n}$ is the heat generation term for the reactions. $h_{i n}$ represents the enthalpy flowrate of the inlet stream at temperature $T_{i n}$ and $h_{\text {out }}$ represents the enthalpy flowrate of the outlet stream at temperature $T_{\text {exit }}$. We consider the case wherein most of the energy carried by the hot stream is transfered to the cold stream and thus a large amount of energy is recycled into the network via the FEHE. We define $\varepsilon$ as the ratio of the energy input through the cold feed to the energy increase of this stream due to the heat transfered from the hot stream i.e., $\varepsilon=\left[F_{\text {in }} \rho c_{p}\left(T_{i n}-T_{\text {ref }}\right)\right]_{s} /\left[F_{\text {in }} \rho c_{p}\left(T_{C, z=0}-T_{i n}\right)\right]_{s}$ where $T_{\text {ref }}$ is the reference temperature. In order to facilitate such a large recovery of heat, the FEHE must provide a sufficiently large heat transfer area. Specifically, from the energy balance on the cold stream of the FEHE, at steady state, we have $\left[U A\left(T_{H, a v}-T_{C, a v}\right)\right]_{s}=$ $\left[F_{i n} \rho c_{p}\left(T_{C, z=0}-T_{i n}\right)\right]_{s}$ which leads to $U A / F_{i n, s} \rho c_{p}=$ $k(\mathcal{O}(1)) / \varepsilon\left(T_{H, a v}\right.$ and $T_{C, a v}$ are the average temperatures for the hot and the cold leg of the FEHE). We define the $\mathcal{O}(1)$ ratios $k_{r}=\left[F_{i n} \rho c_{p}\left(T_{R}-T_{r e f}\right)\right]_{s} /\left[F_{i n} \rho c_{p}\left(T_{C, z=0}-T_{i n}\right)\right]_{s}$, $k_{c}=\left[F_{i n} \rho c_{p}\left(T_{C, z=0}-T_{r e f}\right)\right]_{s} /\left[F_{i n} \rho c_{p}\left(T_{C, z=0}-T_{i n}\right)\right]_{s}$, $k_{f}=\left[F_{i n} \rho c_{p}\left(T_{i}-T_{r e f}\right)\right]_{s} /\left[F_{i n} \rho c_{p}\left(T_{C, z=0}-T_{i n}\right)\right]_{s}$, $u_{r}=\left[F_{i n} \rho c_{p}\left(T_{R}-T_{\text {ref }}\right)\right] /\left[F_{i n} \rho c_{p}\left(T_{R}-T_{\text {ref }}\right)\right]_{s}$, $u_{c}=\left[F_{i n} \rho c_{p}\left(T_{C, z=0}-T_{r e f}\right)\right] /\left[F_{i n} \rho c_{p}\left(T_{C, z=0}-T_{r e f}\right)\right]_{s}$ and $u_{f}=\left[F_{i n} \rho c_{p}\left(T_{i}-T_{\text {ref }}\right)\right] /\left[F_{i n} \rho c_{p}\left(T_{i}-T_{\text {ref }}\right)\right]_{s}$. The energy dynamics equations in Eq. (8) thus become:

$$
\begin{aligned}
\frac{d T_{R}}{d t} & =\frac{\left[F_{i n}\left(T_{i n}-T_{r e f}\right)\right]_{s}}{V}\left[\frac{k_{f} u_{f}-k_{r} u_{r}}{\varepsilon}\right]-\frac{\Delta H^{T} \underline{r}}{\rho c_{p}} \\
\frac{d T_{i}}{d t} & =\frac{\left[F_{i n}\left(T_{i n}-T_{r e f}\right)\right]_{s}}{V_{f}}\left[\frac{k_{c} u_{c}-k_{f} u_{f}}{\varepsilon}\right]+\frac{Q{ }_{H}}{\rho c_{p} V_{f}} \\
\frac{\partial T_{H}}{\partial t} & =-v_{H} \frac{\partial T_{H}}{\partial z}-\frac{k F_{i n, s}}{\varepsilon}\left(\frac{T_{H}-T_{C}}{V_{H}}\right) \\
\frac{\partial T_{C}}{\partial t} & =v_{C} \frac{\partial T_{C}}{\partial z}+\frac{k F_{i n, s}}{\varepsilon}\left(\frac{T_{H}-T_{C}}{V_{C}}\right)
\end{aligned}
$$

Lets now apply the results derived in section II to the model (10). As shown in section II, the enthalpies $H_{c}, H_{h}$ and $H_{r}$ have a component in the fast time scale. These enthalpies correspond to the temperatures of the cold and hot leg of the FEHE and the reactor respectively. The network exit temperature $T_{\text {exit }}$ is related to the temperature of the hot leg FEHE and the reactor temperature by $\alpha^{1}$. So, we can address the control of $T_{\text {exit }}$ in the fast time scale using $\alpha$ as the manipulated input to reject the disturbances affecting the FEHE dynamics (e.g., $\left.T_{i n}\right)$. This is consistent with the observation in remark II.2 regarding manipulating the large internal flows to address the unit level control objectives.

Considering the dynamics in Eq. (10) in the original time scale and taking the limit $\epsilon \rightarrow 0$, we obtain the quasi steady state constraints:

\footnotetext{
${ }^{1}$ Here the bypass ratio $\alpha$ is assumed to be small, which is consistent with
} the assumption of a high rate of energy recovery and recycle. 
TABLE 1

NOMINAL VALUES OF PROCESS PARAMETERS

\begin{tabular}{|c|c||c|c|}
\hline Parameter & Value & Parameter & Value \\
\hline$c_{A o}$ & $1000 \mathrm{~mol} / \mathrm{m}^{3}$ & $k_{o}$ & $1.2667 \times 10^{7} \mathrm{~s}^{-1}$ \\
$T_{i n}$ & $300 \mathrm{~K}$ & $E$ & $142870 \mathrm{~J} / \mathrm{mol}$ \\
$c_{A}$ & $54.79 \mathrm{~mol} / \mathrm{m}^{3}$ & $\Delta H$ & $-54.828 \mathrm{KJ} / \mathrm{mol}$ \\
$T_{R}$ & $922.39 \mathrm{~K}$ & $U A$ & $83680 \mathrm{~W} / \mathrm{K}$ \\
$T_{\text {exit }}$ & $364.86 \mathrm{~K}$ & $\rho c_{p}$ & $4.184 \times 10^{6} \mathrm{~J} / \mathrm{m}^{3} / \mathrm{K}$ \\
$T_{i}$ & $910 \mathrm{~K}$ & $F_{i n}$ & $5.7667 \times 10^{-4} \mathrm{~m}^{3} / \mathrm{s}$ \\
$T_{C, z=0}$ & $873.48 \mathrm{~K}$ & $F$ & $5.7667 \times 10^{-4} \mathrm{~m}^{3} / \mathrm{s}$ \\
$Q_{H}$ & $8.82 \times 10^{4} \mathrm{~W}$ & $V$ & $0.1 \mathrm{~m}^{3}$ \\
$\alpha$ & 0.1 & $V_{H}$ & $0.1 \mathrm{~m}^{3}$ \\
$L$ & $1 \mathrm{~m}$ & $V_{C}$ & $0.09 \mathrm{~m}^{3}$ \\
$\varepsilon$ & 0.0471 & $V_{f}$ & $0.01 \mathrm{~m}^{3}$ \\
\hline
\end{tabular}

$$
\begin{aligned}
0 & =k_{f} u_{f}-k_{r} u_{r} \text { i.e., } T_{i}=T_{R} \\
0 & =k_{c} u_{c}-k_{f} u_{f} i . e .,\left.T_{C}\right|_{z=0}=T_{i} \\
0 & =-k F_{i n, s}\left(T_{H}-T_{C}\right) / V_{H} \\
0 & =k F_{i n, s}\left(T_{H}-T_{C}\right) / V_{C}
\end{aligned}
$$

The last two constraints are not linearly independent and lead to $T_{H}(z, t)=T_{C}(z, t)$ which is a thermal equilibrium condition. We now define the total network enthalpy as:

$$
H_{\text {total }}=\rho c_{p}\left(V_{H} T_{H}+V_{C} T_{C}+V_{f} T_{i}+V T_{R}\right)
$$

The dynamics of the network in the slow time scale is then given by:

$$
\begin{aligned}
d V / d t & =F_{\text {in }}-F \\
d \underline{C} / d t & =F_{\text {in }}\left(\underline{C}_{o}-\underline{C}\right) / V-\underline{\underline{S}}\left(\underline{C}, H_{\text {total }}\right) \\
d H_{\text {total }} / d t & =-\underline{H}^{T} \underline{r}\left(\underline{C}, H_{\text {total }}\right)+Q_{H} \\
0 & =T_{H}-T_{C} \\
0 & =\left.T_{C}\right|_{z=0}-T_{i} \\
0 & =T_{i}-T_{R}
\end{aligned}
$$

Note that the energy dynamics in the slow time scale is one dimensional as predicted in section II. We will illustrate these concepts through a simulation example.

\section{Simulation Results}

We consider a case where a first order irreversible exothermic reaction $A \rightarrow B$ is carried out in the CSTR shown in Fig. 3. The reactant $\mathrm{A}$ is fed to the network at concentration $c_{A o}$ and at temperature $T_{i n}$. The control objectives are to control the outlet concentration of the reactor i.e., $c_{A}$, the network exit temperature i.e., $T_{\text {exit }}$ and the network holdup. The nominal values of the state variables and the process parameters are given in Table 1

The dynamics of this network will be in the form of Eq. (8). The partial differential equations were discretized by a finite difference approximation (taking 100 equidistant nodes) and the resulting ODE system was solved using MATLAB's ODE solver for stiff differential equations. Fig. 4 shows the various energy flows in the network at the steady state. We see that the internal energy flows have higher order of magnitude as compared to the external energy flows. Fig.

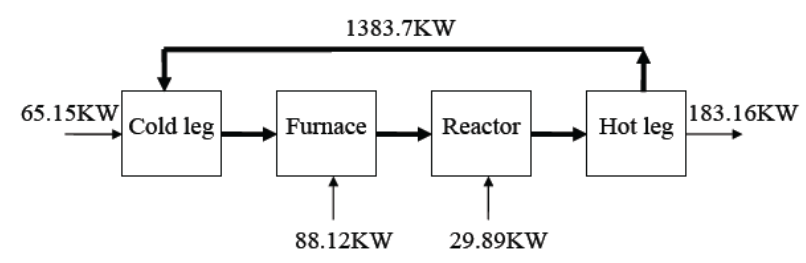

Fig. 4. Steady state energy flows

5 shows the evolution of hot and cold leg exit temperatures of the FEHE, for a $1 \%$ perturbation from the steady state. The plots show a two time scale behavior with a fast transient behavior followed by a slow dynamics. The evolution of the total enthalpy of the network for the same perturbed system, confirms its evolution in the slow time scale.

We address the control of $T_{\text {exit }}$ in the fast time scale using a simple PI control law with $\alpha_{o}=0.1, K_{c}=0.0018 K^{-1}$ and $\tau_{I}=10 \mathrm{~s}$.

$\alpha=\alpha_{o}+K_{c}\left[\left(T_{\text {exit }, s p}-T_{\text {exit }}\right)+\frac{1}{\tau_{I}} \int_{0}^{t}\left(T_{\text {exit }, s p}-T_{\text {exit }}\right) d \tau\right]$

We next proceed to the control objectives in the slow time scale. We have to control $c_{A}$ and $V\left(y_{2}\right)$. Control of concentration, in this case, is equivalent to the control of reactor temperature and so, we control $T_{R}\left(y_{1}\right)$ instead of $c_{A}$. To derive the controllers, we require ODE description of the model (12). With the slow energy dynamics expressed in terms of $T_{R}$ (rather than the overall network enthalpy) the "algebraic" variables are:

$\underline{z}=\left[\begin{array}{c}z_{1} \\ z_{2} \\ z_{3}\end{array}\right]=\left[\begin{array}{c}{\left[F_{\text {in }} / V\left(T_{\text {in }}-T_{r e f}\right)\right]_{s} \lim _{\varepsilon \rightarrow 0}\left(k_{f} u_{f}-k_{r} u_{r}\right) / \varepsilon} \\ {\left[F_{\text {in }} / V_{f}\left(T_{i n}-T_{r e f}\right)\right]_{s} \lim _{\varepsilon \rightarrow 0}\left(k_{c} u_{c}-k_{f} u_{f}\right) / \varepsilon} \\ k F_{i n} / V_{H} \lim _{\varepsilon \rightarrow 0}\left(T_{H}-T_{C}\right) / \varepsilon\end{array}\right]$

The quasi steady state constraints are differentiated to get ODE description of the slow dynamics, described by (13).

$$
\begin{aligned}
\frac{d V}{d t} & =F_{i n}-F \\
\frac{d c_{A}}{d t} & =\frac{F_{i n}\left(c_{A o}-c_{A}\right)}{V}-k_{o} e^{\left(E / R T_{R}\right)} c_{A} \\
\frac{d T_{R}}{d t} & =z_{1}-\left(\Delta H / \rho c_{p}\right) k_{o} e^{\left(E / R T_{R}\right)} c_{A} \\
z_{1} & =z_{2}+\left(\Delta H / \rho c_{p}\right) k_{o} e^{\left.E / R T_{R}\right)} c_{A}+\frac{Q_{H}}{\rho c_{p} V_{f}} \\
z_{2} & =-\frac{k}{V_{C}}\left(V z_{1}+V_{f} z_{2}\right)-\frac{Q_{H}}{\rho c_{p} V_{f}} \\
z_{3} & =0
\end{aligned}
$$

The available manipulated inputs are $Q_{H}\left(u_{1}\right)$ and $F\left(u_{2}\right)$. From Eq. (13), the relative degree vector for this system is $\left[\begin{array}{ll}r_{1} & r_{2}\end{array}\right]=\left[\begin{array}{ll}1 & 1\end{array}\right]$. So, using [13], we design an input/output linearizing controller to induce first order responses in $T_{R}$ and $V$.

$$
\begin{aligned}
\beta_{1,1}\left(d T_{R} / d t\right)+\beta_{1,0} T_{R} & =v_{1} \\
\beta_{2,1}(d V / d t)+\beta_{2,0} V & =v_{2}
\end{aligned}
$$




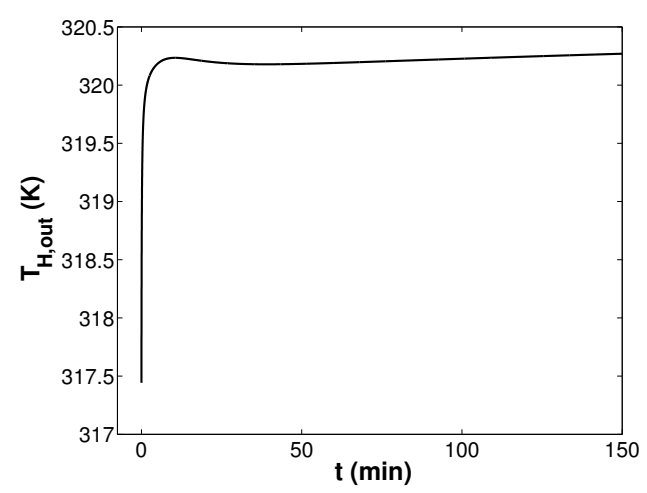

(a) Hot leg of FEHE

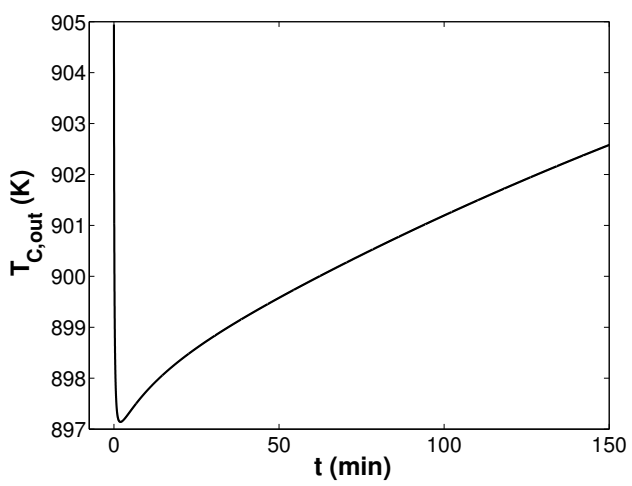

(b) Cold leg of FEHE

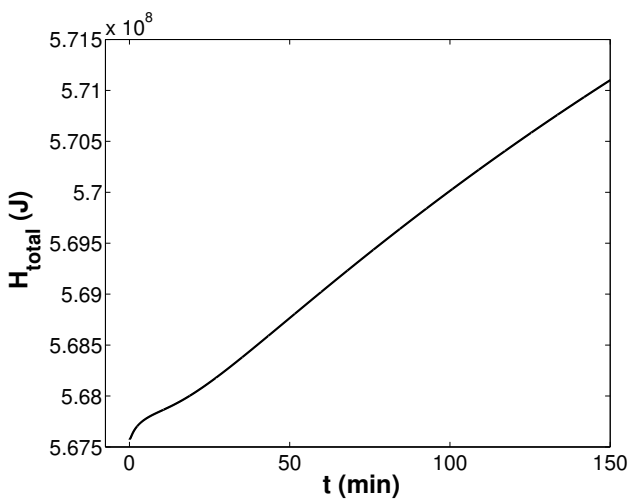

(c) Total enthalpy

Fig. 5. Evolution of FEHE exit temperatures and the network enthalpy for the perturbed system

with, $\beta_{1,1}=10 \mathrm{~s}, \beta_{1,0}=0.001, \beta_{2,1}=20 \mathrm{~s}$ and $\beta_{2,0}=0.002$. To get an offset free response, we used external PI controllers with, $K_{C, 1}=0.008, \tau_{I, 1}=950 \mathrm{~s}, K_{C, 2}=0.01$ and $\tau_{I, 2}=1100 \mathrm{~s}$.

\section{A. Results}

We first test the performance of this controller in the presence of disturbances and modeling errors. We consider that there is $+10 \%$ error in $\Delta H$. The corresponding response in the presence of unmeasured disturbances is shown in Fig. 6. We see that the proposed controller gives good performance in rejecting the disturbance even in the presence of large modeling errors, indicating that the controller is well conditioned. We also note the time scales on which the controllers operate i.e., the $T_{\text {exit }}$ is controlled in the fast time scale while $T_{R}$ is controlled over a slower time horizon.

In the next simulation run, the output tracking performance of the controller is studied for $+5 \%$ change in the set point of $T_{R}$, in the presence of unmeasured disturbance. The corresponding results are shown in Fig. 7. We see that the controller derived based on the reduced order model shows excellent performance in tracking the desired trajectory.

\section{COnClusions}

In this paper, we analyzed networks of reactor and FEHE with tight energy integration. We showed that the dynamics of this network possesses stiffness and shows a two time scale behavior. Using singular perturbation analysis, we derived the reduced order models for the network in each time scale, and we identified the control objectives and the handles (manipulated inputs) available in each time scale. We demonstrated the derived results through a simulation case study. The controllers derived on the basis of the reduced order models are well conditioned and show good output tracking performance.

References are important to the reader; therefore, each citation must be complete and correct. If at all possible, references should be commonly available publications.

\section{REFERENCES}

[1] J.M. Douglas, Conceptual Design of Chemical Processes, McgrawHill; 1988.

[2] C.S. Bildea and A.C. Dimian, "Stability and multiplicity approach to the design of heat integrated pfr", AIChE J., vol. 44, 1988, pp 27032712.

[3] Y.H. Chen and C.C. Yu, "Design and control of heat integrated reactors",Ind. Eng. Chem. Res., vol. 42, 2003, pp. 2791-2808.

[4] J.C. Morud and S. Skogested, "Dynamic behavior of integrated plants", J. Proc. Contr., vol. 6, 1996, pp. 145.

[5] F. Reyes and W.L. Luyben, "Steady-state and dynamic effects of design alternatives in heat-exchanger/furnace/reactor processes", Ind. Eng. Chem. Res., vol. 39, 2000, pp. 3335-3346.

[6] W.L. Luyben, "External versus internal open loop unstable processes", Ind. Eng. Chem. Res., vol. 37, 1998, pp. 2713-2720.

[7] B.D. Tyreus and W.L. Luyben, "Unusual dyanmics of a reactor/preheater process with deadtime, inverse response and openloop instability", J. Proc. Contr., vol. 3, 1993, pp. 241-251.

[8] C.S. Bildea, A.C. Dimian, and P.D. Iedema, "Multiplicity and stability approach to the design of heat integrated multibed plug flow reactor", Comput. Chem. Eng., vol. 25, 2001, pp. 41-48.

[9] W.L. Luyben, "Effect of design and kinetic parameters on the control of cooled tubular reactor systems", Ind. Eng. Chem. Res., vol.40, 2001, pp. 3623-3633.

[10] Y.H. Chen and C.C. Yu, "Interaction between thermal efficiency and dynamic controllability for heat integrated reactors" Comput. Chem. Eng., vol.24, 2000, pp. 1077-1082.

[11] M. Baldea and P. Daoutidis, "Model reduction and control of reactorheat exchanger networks", J. Proc. Contr., vol. 16, 2006, pp. 265-274.

[12] A. Kumar and P. Daoutidis, "Nonlinear dynamics and control of process systems with recycle", J. Proc. Contr., vol. 12, 2002, pp. 475484.

[13] A. Isidori, Nonlinear Control Systems, Springer, London; 1995. 


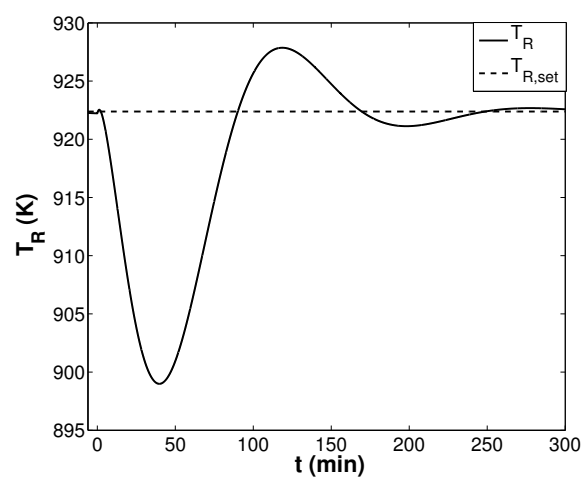

(a) Reactor temperature

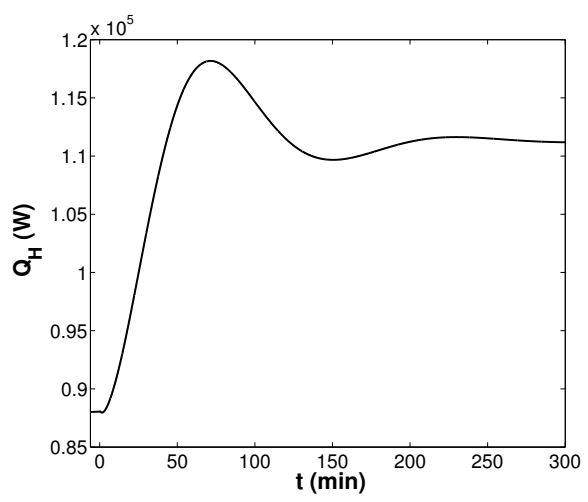

(b) Furnace duty

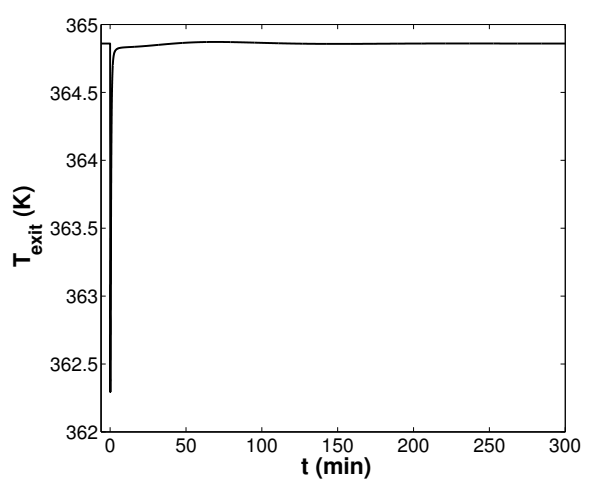

(c) Network exit temperature

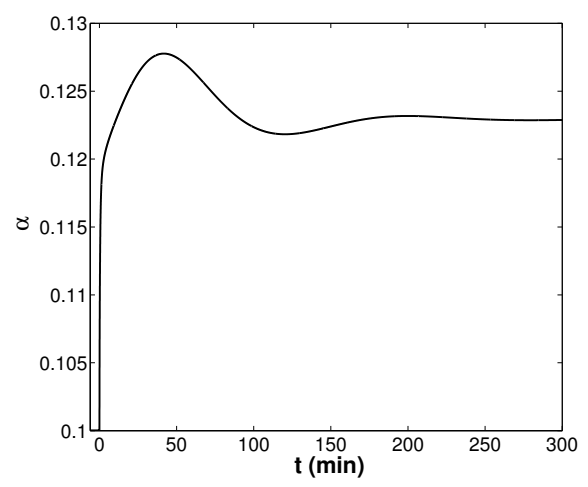

(d) Bypass ratio

Fig. 6. Closed loop response in the presence of $+10 \%$ modeling error in $\Delta H$ and unmeasured disturbances

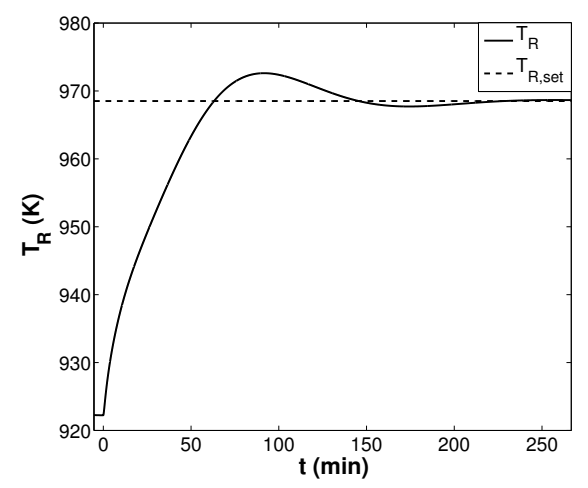

(a) Reactor temperature

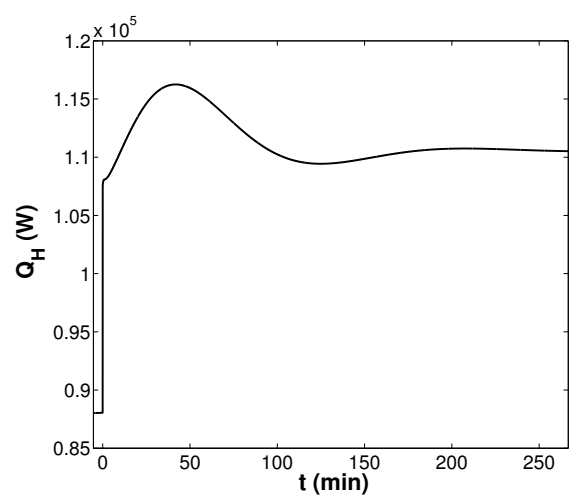

(b) Furnace duty

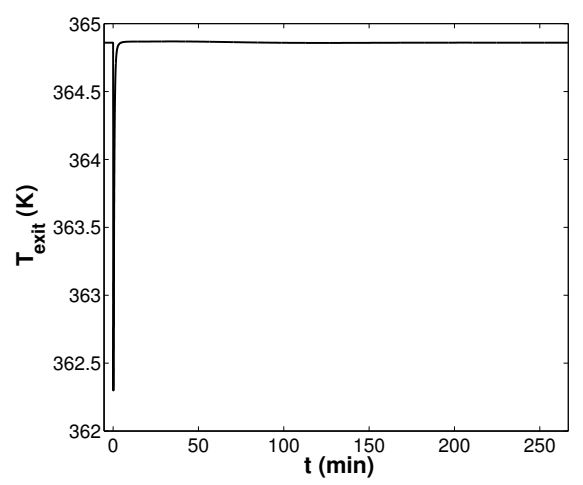

(c) Network exit temperature

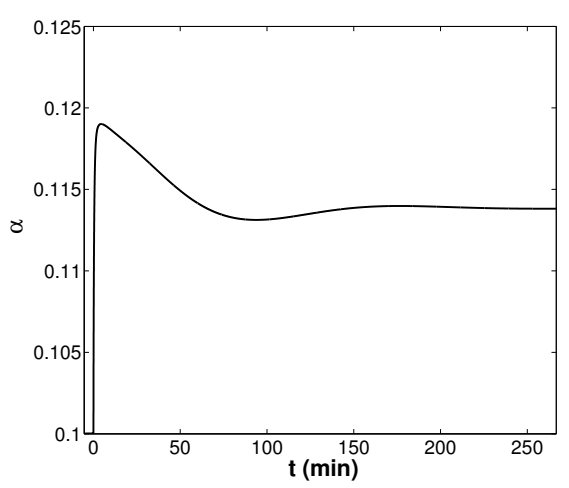

(d) Bypass ratio

Fig. 7. Closed loop response for $+5 \%$ change in $T_{R, \text { set }}$, in the presence of $+10 \%$ modeling error in $\Delta H$ and unmeasured disturbances 